\title{
Molecular Cytogenetic Analysis of Cervical Squamous Cell Carcinoma Cells Demonstrates Discordant Levels of Numerical and Structural Chromosomal Instability and Identifies 'Selected' Chromosome Rearrangements
}

\author{
N. Foster S. Carter G. Ng M. Pett I. Roberts N. Coleman \\ Medical Research Council Cancer Cell Unit, Hutchison/MRC Centre, Cambridge, UK
}

\section{Key Words}

Carcinoma $\cdot$ Cervix $\cdot$ Chromosomal instability $\cdot$

Rearrangements $\cdot$ Selection

\begin{abstract}
The processes underlying the karyotype complexity and heterogeneity characteristic of cervical squamous cell carcinoma (SCC) cells are not well understood, nor is it known which chromosome abnormalities provide a selective advantage. We used molecular cytogenetics to quantify for the first time the prevalence of all chromosomal abnormalities detectable in 8 cervical SCC cell lines. Of 225 rearrangements identified, 118 occurred frequently ( $\geq 70 \%$ metaphases) in one or more lines, indicating selection. Most of these selected rearrangements $(107 ; 48 \%$ of the total) were included in published lists of abnormalities detected in independent cell stocks (although their significance was not previously recognised), indicating that they arose early in the establishment of the lines and were therefore most likely to have been present in the original tumours. The 8 cell lines showed 4.7- and 11.0-fold variation in levels of numerical and structural chromosomal instability ( $\mathrm{N}-\mathrm{CIN}$ and $\mathrm{S}-\mathrm{CIN}$ ) respective$\mathrm{ly}$, as determined by quantifying non-clonal abnormalities. We observed discordance between N-CIN and S-CIN ( $p=$ 0.148), with modal chromosome number showing a strong
\end{abstract}

association with $\mathrm{N}-\mathrm{CIN}(\mathrm{p}=0.007)$ but not with S-CIN ( $p=$ 0.602). These data imply that different mechanisms underlie $\mathrm{N}-\mathrm{CIN}$ and S-CIN in cervical SCC. Despite ongoing S-CIN, newly arisen in vitro rearrangements were infrequently selected, supporting the view that selection is an important determinant of somatic evolution in malignancy. There was a positive association between numbers of non-clonal and clonal rearrangements ( $p=0.002$ for non-clonal vs. selected rearrangements), suggesting that factors affecting S-CIN determine the total numbers of rearrangements present in established cervical SCC cells.

Copyright $\odot 2010$ S. Karger AG, Base

Cervical malignancy is the second most common cause of cancer deaths in women worldwide [Morris et al., 1996]. Most cases are squamous cell carcinomas (SCCs), which arise in a multistep fashion from precursor squamous intraepithelial lesions [Pett and Coleman, 2007]. Infection with high-risk human papillomavirus (HR-HPV) is considered necessary but insufficient for malignant progression [zur Hausen, 2000], with host genomic instability also thought to be required [Pett et al.,

N.F. and S.C. contributed equally to this study

\section{KARGER}

Fax +4161306 1234

E-Mail karger@karger.ch

www.karger.com (c) 2010 S. Karger AG, Basel

$1424-8581 / 09 / 1271-0009 \$ 26.00 / 0$

Accessible online at:

www.karger.com/cgr
Nicholas Coleman

Medical Research Council Cancer Cell Unit, Hutchison/MRC Centre Cambridge CB20XZ (UK)

Tel. +44 122376 3285, Fax +44 1223763284

E-Mailnc109@hutchison-mrc.cam.ac.uk 
2004; Pett and Coleman, 2007]. Cervical SCC cells are characterised by complex karyotypes [James et al., 1989; Zimonjic et al., 1995; Szuhai et al., 2000; Harris et al., 2003], although the processes that underlie such complexity are not fully understood, and little is known about which genes are most commonly affected. An important strategy for detecting potential 'driver' genes is to identify recurrent chromosomal rearrangements acquired during cervical neoplastic progression. In view of the considerable difficulties in preparing metaphase chromosomes from clinical samples, cell lines have generally been used for this purpose [James et al., 1989; Zimonjic et al., 1995; Szuhai et al., 2000; Harris et al., 2003].

There is growing interest in understanding the complex karyotypic heterogeneity that exists in carcinomas as a result of chromosomal instability (CIN) [Rajagopalan et al., 2003; Duesberg et al., 2006; Heng et al., 2006; Bayani et al., 2007]. The best-described form of CIN is characterised by progressive alterations in chromosome number, resulting in aneuploidy. Development of aneuploidy in carcinomas often involves tetraploidisation of cells (in which numerical and structural chromosomal abnormalities may have already occurred), followed by chromosome loss and/or gains leading to near-tetraploid or near-triploid cells [Heim et al., 1988; Heim and Mitelman, 1995]. Alternatively, numerical abnormalities may occur independently of tetraploidisation [Heim et al., 1988; Heim and Mitelman, 1995]. It is also now recognised that CIN may operate at the level of chromosome structure, leading to rearrangements [Trent et al., 1989; Mitelman et al., 1994]. Indeed, there is evidence for some carcinoma types that levels of numerical and structural instability do not necessarily correlate [Roschke et al., 2002; Gorringe et al., 2005; Bayani et al., 2008], prompting suggestions that separate consideration be given to numerical CIN (N-CIN) and structural CIN (S-CIN) [Bayani et al., 2008]. However, the existing data predominantly concern adenocarcinomas, with virtually no information for SCC cells.

Published reports also make it difficult to identify recurrent chromosomal abnormalities acquired during cervical neoplastic progression, from which 'driver' host genes are most likely to be identified. An early molecular cytogenetic study used the relatively low-resolution techniques of spectral karyotyping (SKY) and metaphasebased comparative genomic hybridisation (mCGH) to derive karyotypes of 8 commonly used cervical SCC cell lines [Harris et al., 2003]. However, this study reported composite karyotypes only (which describe all clonal abnormalities and require structural rearrangements and chromosomal gains to be present in only 2 metaphases and chromosomal losses in only 3 metaphases [ISCN, 2005], without indicating the frequency at which individual chromosomes were detected.

Consequently, available data provide no indication of the relative frequency of individual cytogenetic abnormalities in cervical SCC. In particular, it is not possible to distinguish the abnormalities that are present throughout a population of cells (indicative of a selective advantage that is likely to reflect functional importance) [Heim et al., 1988] from those that are present relatively infrequently. Moreover, there is no record of the frequency of non-clonal abnormalities in the cell lines. Such abnormalities either provide no selective advantage in vitro or were acquired relatively recently and are yet to be selected. Nevertheless, the frequency of non-clonal abnormalities in individual cell lines can be used to quantify prevailing levels of N-CIN and S-CIN [Roschke et al., 2002, 2003; Bayani et al., 2008; Ye et al., 2009].

In the present study we have undertaken detailed karyotypic analysis of the 8 widely available cervical SCC cell lines. We used multiple 'tailored' sets of 7-colour multiplex fluorescence in situ hybridisation (M-FISH) paints [Roberts et al., 1999; Nacheva et al., 2000], together with $1-\mathrm{Mb}$ array CGH (aCGH); techniques that provide greater resolution than SKY/mCGH. We present all available information for each cell line individually, using schematic depictions and maps of translocation breakpoints, and, importantly, give the frequency of all abnormalities detected. We compare our findings with available karyotype information obtained using independent stocks of the same cell lines [Harris et al., 2003], in order to identify abnormalities that remain common to different stocks, despite the karyotype divergence that would be predicted to occur in different in vitro cultures [as observed in 2 SKY analyses of a single cervical SCC cell line, SiHa, Szuhai et al., 2000; Harris et al., 2003].

Our approach allowed us to address 2 major aims. First, we sought to identify for each cell line the frequently occurring (i.e. selected) rearrangements that are most likely to be of biological significance. We further reasoned that the selected rearrangements that were also observed in independent cell stocks by SKY [Harris et al., 2003] must have arisen early in the evolution of the cell lines and would therefore be most likely to include abnormalities present in the tumour samples from which the lines arose. Second, we used information on cell-to-cell karyotype variation to determine prevailing rates of $\mathrm{N}$ $\mathrm{CIN}$ and S-CIN and investigate relations between them and with the modal chromosome number. 


\section{Materials and Methods}

\section{Cell Lines}

The cervical SCC cell lines studied were: C-33A, C-4I, CaSki, HT-3, ME180, MS751, SiHa and SW756 (online supplementary table S1; for all online supplementary material see www. karger.com/doi/10.1159/000290954). All of them were obtained from the American Type Culture Collection and grown in continuous culture for no more than one month. Genomic DNA and metaphase spreads were prepared as described [Roberts et al., 1999].

\section{Molecular Cytogenetic Analysis and Assessment of CIN}

Full details of the aCGH profiling have been given previously [Ng et al., 2007]. The arrays used represented 4,134 bacterial artificial chromosome (BAC) clones with a mean gap size of $0.92 \mathrm{Mb}$. Fluorescence ratios were calculated as the average of paired dyeswapped arrays.

M-FISH was performed using multiple 'tailored' sets of 7-colour paints, designed to identify translocations of interest at improved resolution [Roberts et al., 1999]. The imaging system used for M-FISH gives greater cytogenetic information than an MkI Spectral-Cube, by providing a larger CCD array and a smaller pixel size, which improves the ability to discern chromosome bands [Garini et al., 1996]. The first paint sets generated for each cell line were based on the published karyotypes available at the time of analysis [James et al., 1989; Zimonjic et al., 1995; Szuhai et al., 2000]. Where individual rearrangements were not conclusively identified from the first paint sets, further sets were prepared. This process continued iteratively until all chromosome abnormalities had been analysed. Wherever possible, chromosomes potentially involved in rearrangements were painted in contrasting colours, in order to make reliable identification easier.

Total chromosome numbers were determined in 50 metaphases of each cell line. The frequency of each abnormality was determined in 10 metaphases labelled with a paint set that enabled clear identification of the relevant chromosome(s). In total, 5 or 6 7-colour paint sets (50-60 metaphases) were required to obtain detailed karyotypes for each cell line. Representative composite karyotypes were derived by combining information on ploidy levels and all painting data [ISCN, 2005]. A rearrangement was defined by us as occurring frequently (thereby implying it conferred a selective advantage) if it was seen in $\geq 70 \%$ metaphases examined.

Breakpoints of detectable rearrangements (translocations, deletions, insertions, duplications, inversions and homogenously staining regions) were assigned, where possible, using aCGH data and inverted enhanced DAPI images. In keeping with previous studies, we defined recurrent breakpoints as those that occurred in 2 or more derivative chromosomes [Rao et al., 1998] and breakpoint clusters as those that occurred in 4 or more derivative chromosomes [Harris et al., 2003]. For duplicated derivative chromosomes and reciprocal translocations, breakpoints were counted only once.

$\mathrm{N}$-CIN levels were determined using an adaptation of previously published methods [Lengauer et al., 1997; Bayani et al., 2008]. The availability of metaphases enabled us to identify numerical abnormalities of all assessable chromosomes, rather than the subset of chromosomes that would be analysed using inter-

Chromosomal Instability in Cervical

SCC phase FISH. For each chromosome we first established the modal number (counting centromeres) and then determined the number of cells in which numbers of that chromosome deviated from the mode (either greater or lower; and regardless of the size of the deviation), using 10 appropriately painted metaphases in all cases. If the same non-modal number of morphologically identical chromosomes was seen in greater than $30 \%$ of cells, such cells were considered to represent a clone and were not counted [Bayani et al., 2008]. If no modal number could be determined for a particular chromosome (due to equal representation of different numbers of that chromosome in the cells examined), it was excluded. N-CIN levels in each cell line were determined from the total number of deviations from modal chromosome numbers, normalised for how many of the 23 different chromosomes could be assessed, to generate an N-CIN index. In total, it was possible to assess numerical variations of 153 chromosomes (each in 10 appropriately painted metaphases) across the 8 cell lines.

$\mathrm{S}$-CIN indices were obtained by determining the numbers of non-clonal rearrangements. As clonal rearrangements are defined as those present in 2 or more cells [ISCN, 2005], non-clonal abnormalities are those present in 1 metaphase only (equating to $10 \%$ of the 10 metaphases examined with the appropriate paint set). Associations between S-CIN, N-CIN and ploidy were examined statistically using Pearson's correlation and basic linear regression. All p values were 2-tailed.

\section{Results}

\section{Chromosomal Abnormalities in Cervical SCC Cells}

Using 'tailored' M-FISH and aCGH, we identified 308 clonal abnormalities equivalent to those included in a composite karyotype. Representative M-FISH images are shown in figure 1 , while the aCGH data is shown for each cell line individually in online supplementary figure S1. The clonal abnormalities included 182 rearrangements, of which 118 were frequently occurring ( $\geq 70 \%$ metaphases), indicating selection. Derived composite karyotypes are given in online supplementary table S2, while figure 2 depicts all relevant chromosomes (including the frequency of occurrence of each), using schematics that enable comparisons to be made within and between cell lines. In addition, we identified 43 rearrangements that were non-clonal (i.e. present in a single metaphase only), making 225 rearrangements identified in total (online suppl. table S3).

The cells showed considerable variation in total numbers of rearrangements, which ranged from 9 in C-33A to 50 in HT-3. The number of selected rearrangements also varied considerably, from 7 in C-33A to 27 in CaSki. The vast majority of translocations were unbalanced. There was only 1 dicentric chromosome and no multicentric or ring chromosomes. The non-clonal rearrangements showed no greater complexity than the clonal rearrange- 
A

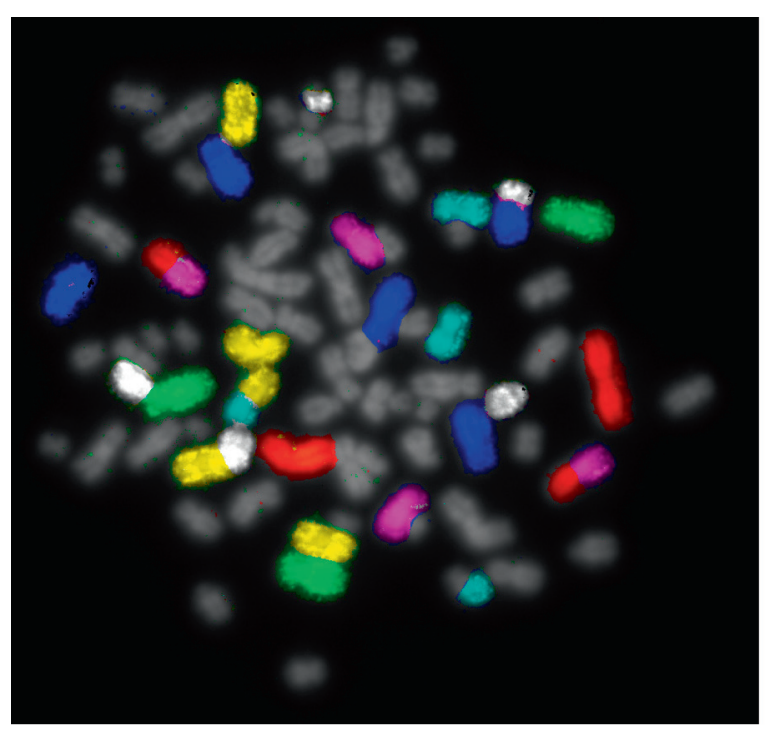

C

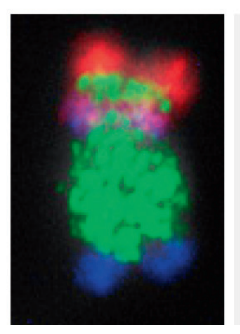

B
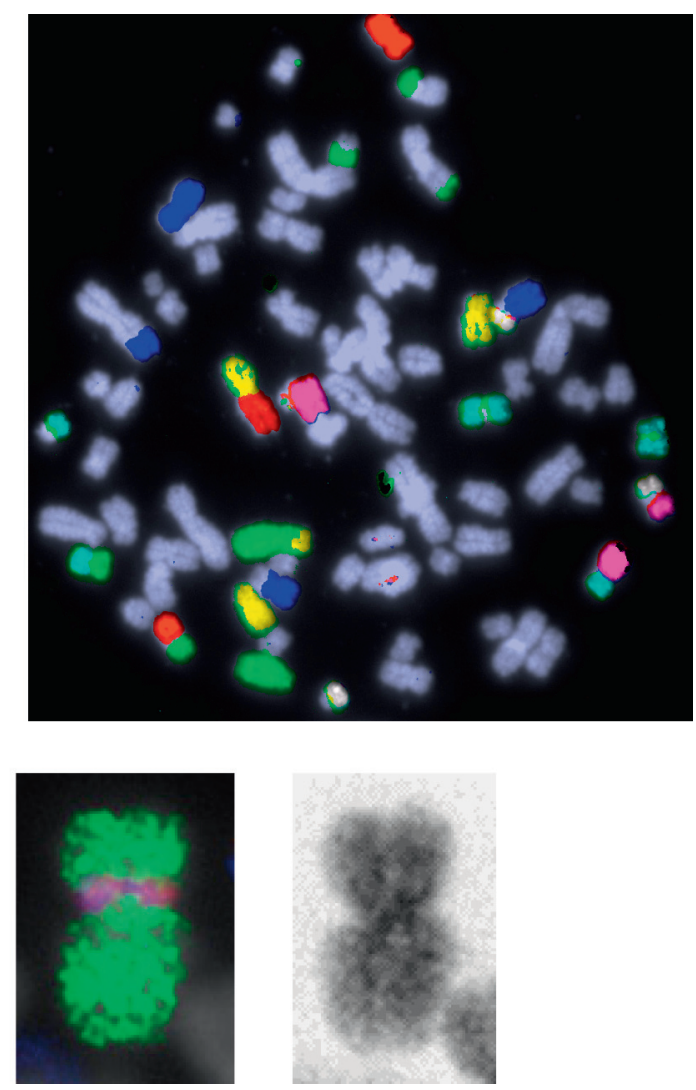

Fig. 1. Identification of chromosome rearrangements by M-FISH. A Representative image obtained following M-FISH pixel classification, using paint set 2 designed for SW756 (red = chromosome 2, green $=4$, blue $=6$, yellow $=7$, magenta $=8$, cyan $=11$, white $=7$ ). $\mathbf{B}$ Representative image obtained using paint set 1 for CaSki $($ red $=X$, green $=5$, blue $=7$, yellow $=8$, magenta $=10$, cyan $=16$, white $=21)$. C Identification of 2 structural abnormalities involving chromosome $X$ in $\mathrm{SiHa}$ : $\operatorname{der}(\mathrm{X}) \mathrm{t}(\mathrm{X} ; 5)$ ins $(\mathrm{X} ; 3) \mathrm{t}(\mathrm{X} ; 12)$ (left) and ins $(\mathrm{X} ; 3)$ (right). In both images red $=5$, green $=X$, blue $=12$, magenta $=3$.

ments. We confirmed that isochromosome $\mathrm{i}(5)(\mathrm{p} 10)$ is a recurrent finding in cervical SCC cells, as it was detectable in 100\% of metaphases of SiHa and SW756 and 90\% of metaphases of C-4I. We also identified a novel reciprocal translocation, $\mathrm{t}(8: 12)(\mathrm{p} 22 ; \mathrm{p} 12)$, which was present in $100 \%$ of metaphases of MS751. It should be noted, however, that no constitutional karyotype is available for the patient from whom this cell line was derived, and so the significance of this translocation remains uncertain.

We assigned 215 breakpoints in rearranged chromosomes, using aCGH copy number karyograms and enhanced inverted DAPI images (fig. 3). A further 124 breakpoints could not be assigned with confidence. Half (108/215) of the assigned breakpoints involved centromeres or pericentromeric bands (p11 or q11). We identified 53 recurrent breakpoints (present in 2 or more de- rivative chromosomes) (online suppl. table S4A), of which 10 (in bold in online suppl. table S4A) were in bands known to contain common fragile sites (www.ncbi.nlm. nih.gov) [Dall et al., 2008]. We also identified 9 breakpoint clusters (present in 4 or more derivative chromosomes), at 5p13, 5p11, 5p10, 7q21, 8q11, 9p11, 14p11, 18q12 and $21 \mathrm{p} 11,3$ of which were in bands containing common fragile sites (5p13, 7q21 and 18q12). Three breakpoint clusters (5p11, 7q21 and 8q11) were present in 4 different cell lines; frequently in $\geq 70 \%$ metaphases, indicating selection.

We compared the 182 clonal rearrangements detected by us with the 181 rearrangements listed in the composite karyotypes obtained by SKY/mCGH [Harris et al., 2003], in order to identify abnormalities common to different cell stocks. Allowing for the lower resolution of the latter 
Fig. 2. Schematic depiction of clonal abnormalities detected. For each chromosome the frequency of occurrence in 10 appropriately painted metaphases is given underneath. The chromosomes are colour coded. Dashed segments represent regions known to be from a particular chromosome but for which breakpoints are unknown. White segments represent unassigned chromosomal material. Where this includes the centromere, the precise origin of the derivative chromosome is not certain. Such chromosomes (8 of the 308 ; $2.6 \%$ ) were assigned to the most likely category, based on the other chromosome material in the derivative.

(For figure 2: CaSki, HT-3, ME180, MS751, $\mathrm{SiHa}$ and SW756 see next pages.)
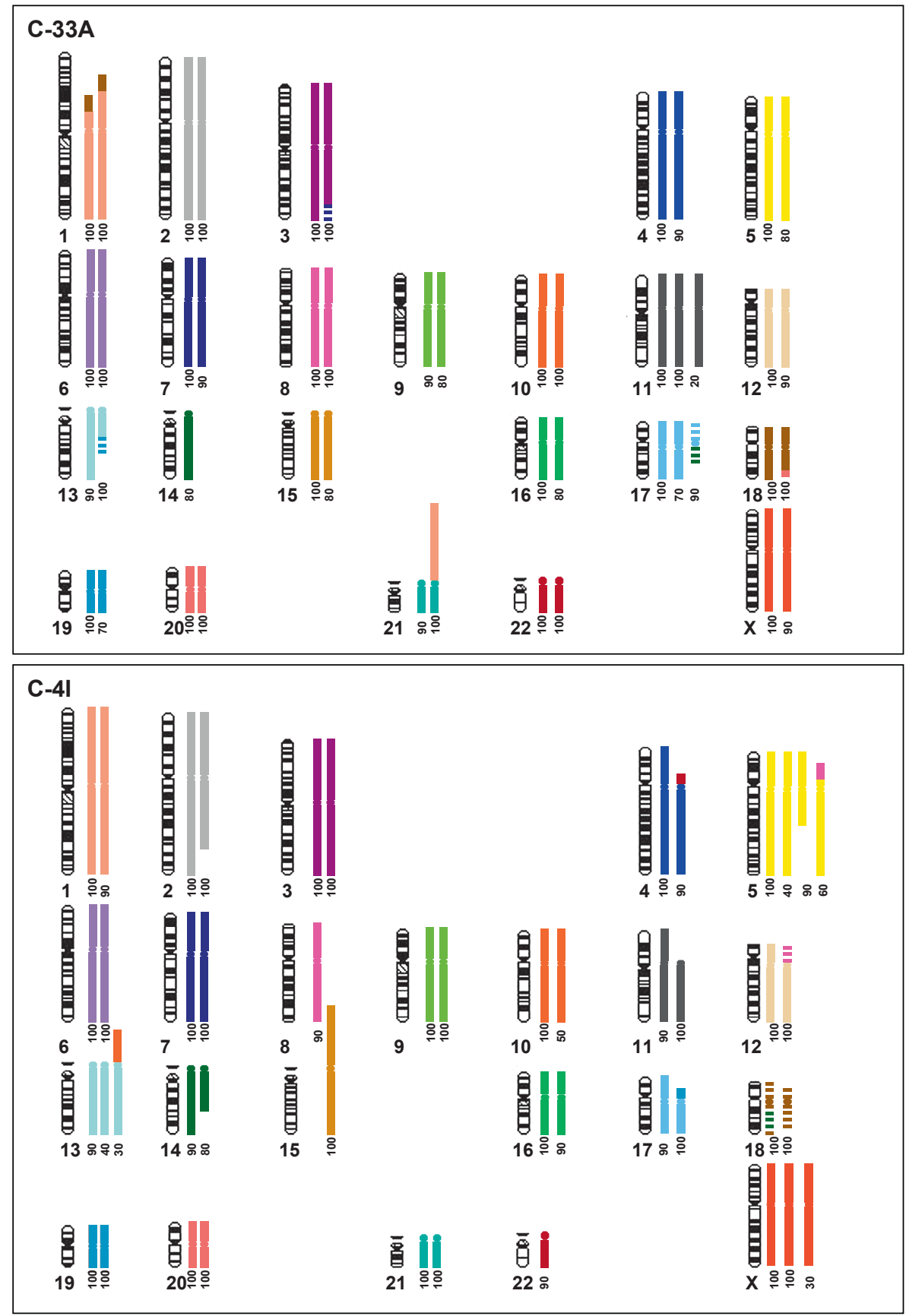

approaches, we consider that 139 of the 182 rearrangements in our composite karyotypes (76\%) were reported in the previous study (online suppl. table S3). The frequency at which rearrangements detected by us in $\geq 70 \%$ metaphases were seen in the SKY/mCGH study (91\%; $107 / 118$ ) was substantially greater than the equivalent frequency for clonal rearrangements detected by us in $\leq 60 \%$ metaphases $(50 \% ; 32 / 64)$. Of the 43 non-clonal rearrange- ments detected by us (i.e. those in $10 \%$ metaphases), only $4(9.3 \%)$ were previously seen by SKY/mCGH. For further comparisons with the previous published study, see online supplementary results.

\section{Levels of Ploidy, N-CIN and S-CIN}

Of the 8 cervical SCC cell lines, 2 were near-diploid (C-33A and C-41). The remainder were aneuploid, being 

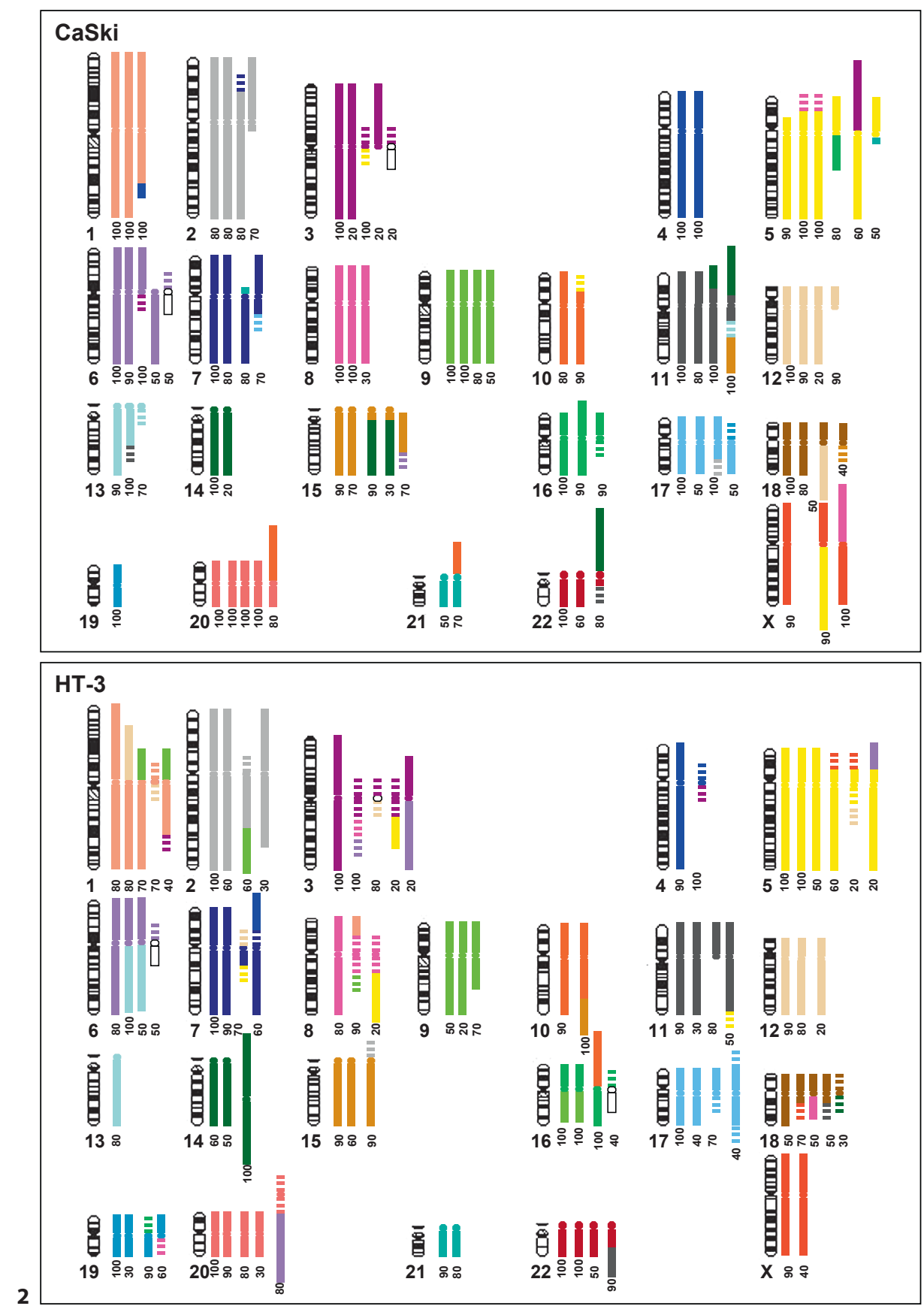

either hypo-tetraploid (HT-3, MS751 and SW756), hypertriploid (SiHa and CaSki) or hypo-triploid (ME180), suggesting progression for most lines via a tetraploid intermediate [Heim and Mitelman, 1995]. In the majority of the aneuploid lines individual structural abnormalities were not duplicated, indicating that they were acquired after the presumed endoreduplication. The exception was MS751, where 9 structural abnormalities were pres- ent in 2 copies. Modal chromosome numbers showed no significant association with the total number of rearrangements $(\mathrm{p}=0.133)$, nor with the number of selected ( $\geq 70 \%$ metaphases) rearrangements $(\mathrm{p}=0.124)$.

The cell lines showed substantial variation in N-CIN rates, with a 4.7-fold difference between the lowest and highest levels (in C-4I and MS751, respectively). N-CIN rates were substantially higher in the aneuploid cells 


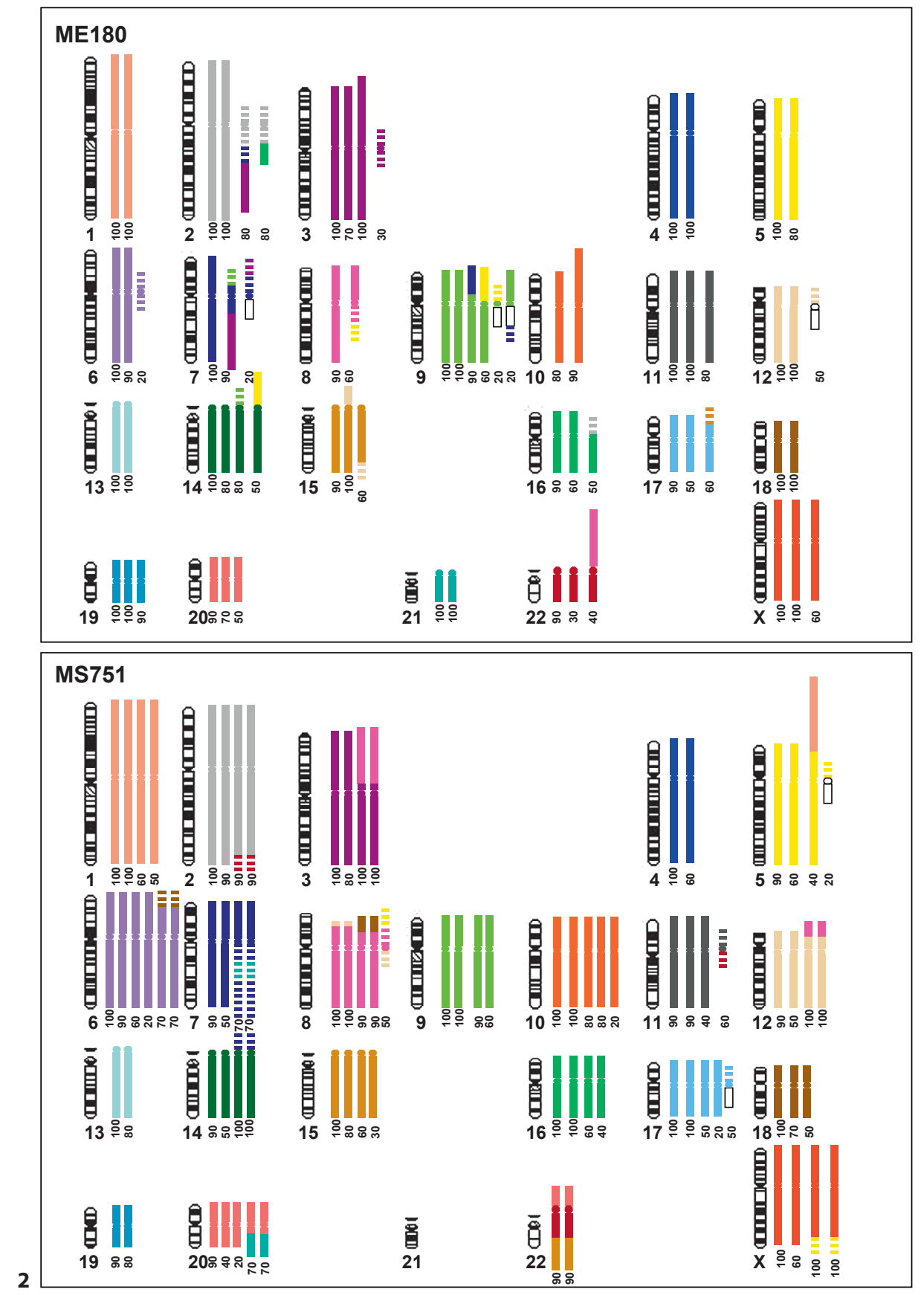

compared to the near-diploid cells, and there was a strong association between N-CIN levels and modal chromosome number ( $\mathrm{p}=0.007$ ) (fig. $4 \mathrm{~A})$. There was also considerable variation in S-CIN rates, with an 11.0-fold difference between the lowest and highest levels (in SW756 and CaSki, respectively). In contrast to N-CIN, however, there was no association between $\mathrm{S}$-CIN and modal chromosome number $(\mathrm{p}=0.602)$ (fig. $4 \mathrm{~B})$.
As an alternative indicator of S-CIN levels, we determined the number of non-clonal rearrangements as a percentage of modal chromosome number, in order to normalise for the number of chromosomes available for rearrangement. We ranked the 8 cell lines according to this value (fig. 4C), for which we observed a 6.1-fold difference between the lowest and highest levels (again in SW756 and CaSki, respectively). Across the cell lines 


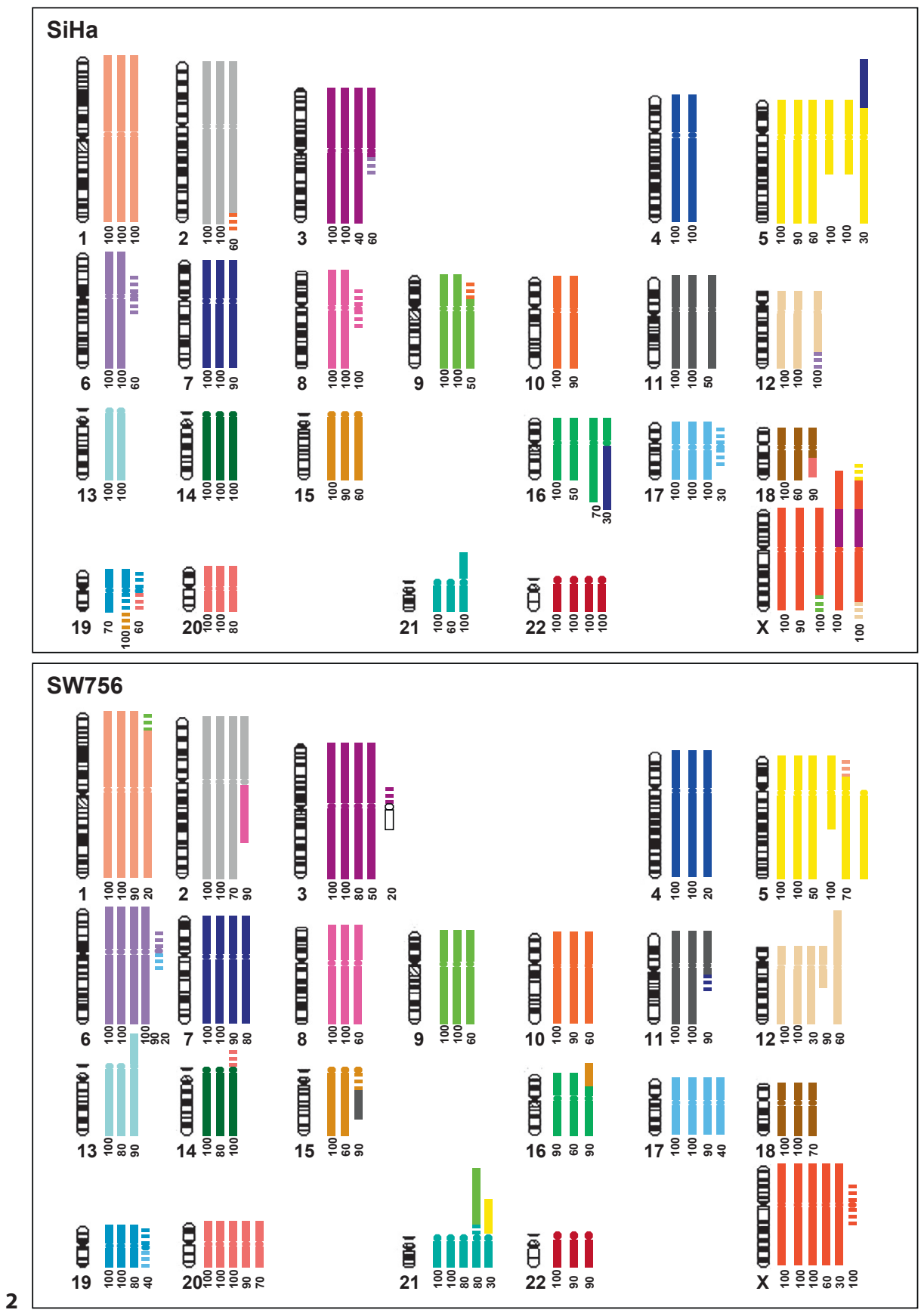

there was no association between N-CIN and S-CIN levels, whether assessing S-CIN by total numbers of nonclonal rearrangements $(\mathrm{p}=0.148)$ or by the normalised values $(p=0.163)$. Interestingly, however, the highest rates of both S-CIN and N-CIN were seen in 3 of the 4 cell lines that originated from metastatic rather than primary cervical SCCs (HT-3, MS751 and CaSki) (fig. 4A and 4B).
Differences between the cell lines in numbers of nonclonal rearrangements (i.e. the S-CIN rates) were generally retained when assessing the numbers of clonal rearrangements present. This was the case for both low-frequency and high-frequency clonal rearrangements, as the relationships between the lines were generally maintained regardless of the frequency threshold required for a particular rearrangement to be counted (across the 


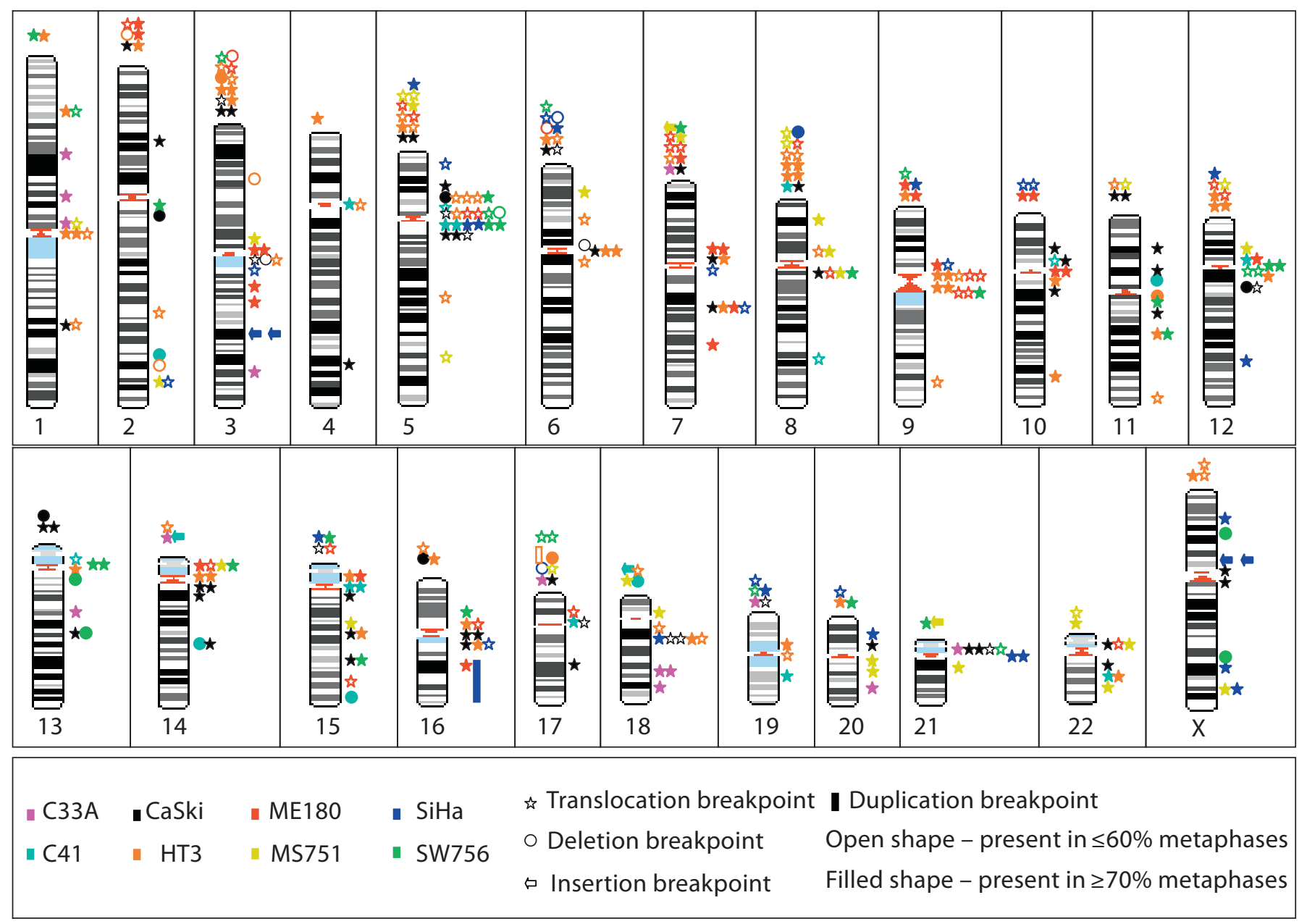

Fig. 3. Location of clonal breakpoints in each cell line. Breakpoints that could not be assigned to a chromosomal band are shown above each chromosome. Data for individual cell lines are colour coded.

range from $10 \%$ to $100 \%$ metaphases) (fig. 4D). While increasing the threshold value (x-axis in fig. 4D) generally led to an increase in the number of abnormalities present in each cell line (y-axis in fig. 4D), the comparative relationships between the lines did not substantially alter. In keeping with these findings, there was a positive correlation across the cell lines between the number of nonclonal rearrangements and the number of selected rearrangements ( $\geq 70 \%$ metaphases; $\mathrm{p}=0.002)$.

When assessing numbers of rearrangements controlled for modal chromosome number, there was a change in the ranking order of the cells (compared to their ranking by total numbers of rearrangements), as would be expected given the lack of association between $\mathrm{S}$-CIN levels and modal chromosome number. However, the differences between the cell lines when assessed in this manner were again generally retained when clonal rearrangements were included, at all frequency thresholds (online suppl. fig. S2).

\section{Discussion}

The analyses presented here were possible because we determined the frequency at which each chromosomal rearrangement was seen in each cell line. One benefit of this approach is that it allowed the identification for each cell line of the frequently occurring (i.e. selected) abnormalities that are most likely to be of importance in cervical carcinogenesis [Heim et al., 1988]. These are listed in online supplementary table S3 (those present at $\geq 70 \%$ frequency). The selected rearrangements seen by us that 


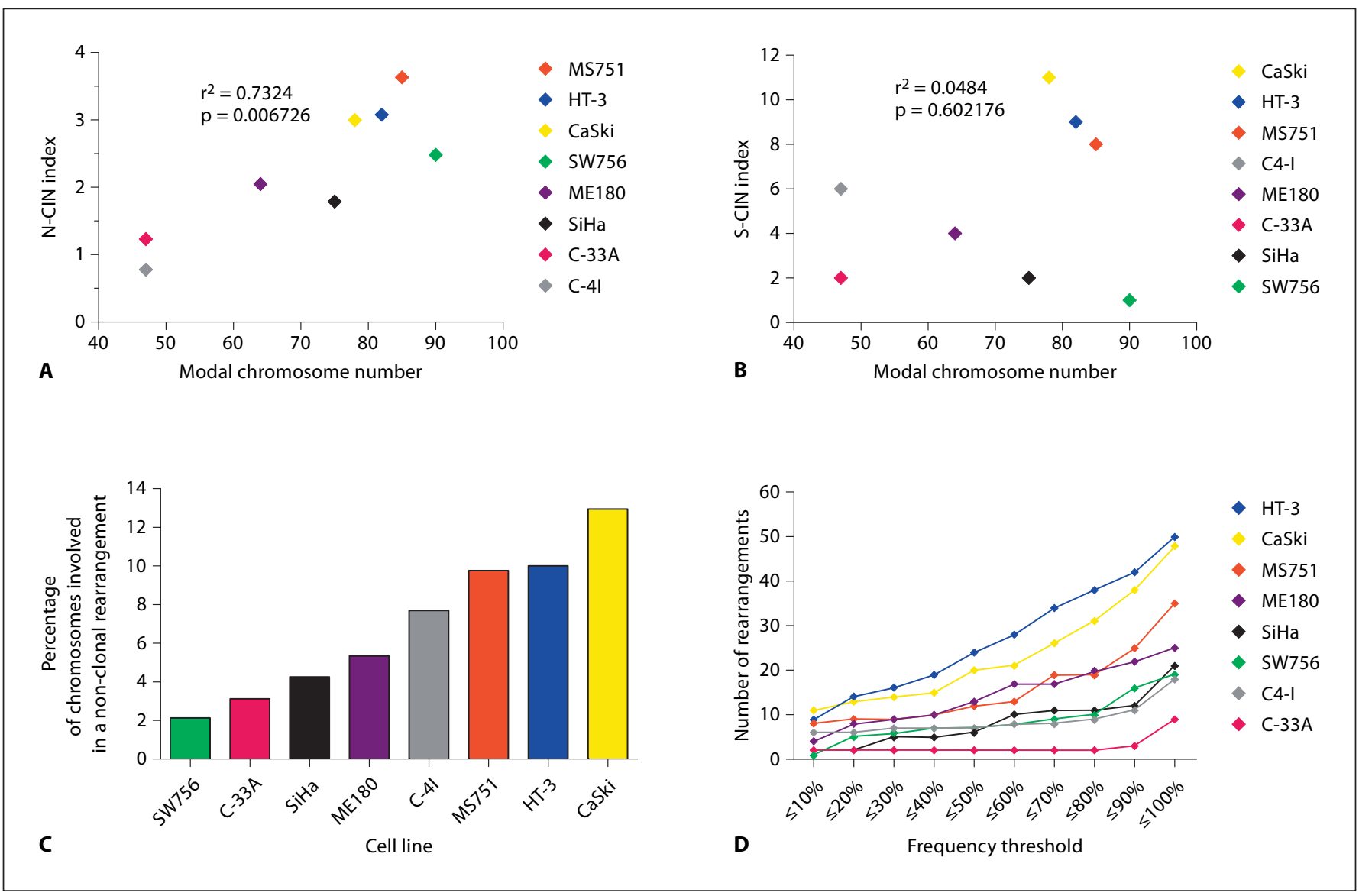

Fig. 4. CIN levels in cervical SCC cells. A, B Across the 8 cell lines the modal chromosome number showed a strong correlation with the N-CIN index (A) but no association with the S-CIN index (B). C There was a wide range in the numbers of non-clonal rearrangements controlled for modal chromosome number, with a greater than 6-fold difference between levels at the extremes. D Differences between the cell lines in the number of non-clonal rear- rangements were generally maintained when numbers of clonal rearrangements were included. The graph plots the prevalence of rearrangements in each cell line (y-axis) against different thresholds of the frequency at which an abnormality was required to be present for inclusion (x-axis). Increasing the frequency threshold generally enabled the inclusion of greater numbers of rearrangements, hence the upward slope of most curves. were also identified in independent cell stocks by SKY/ mCGH [Harris et al., 2003] must have arisen early in the evolution of the cell lines, either in vivo or in the early stages of cell line establishment in vitro. Indeed, many of the frequently occurring (selected) abnormalities seen by us were also described in other, earlier reports using lower resolution cytogenetic techniques [James et al., 1989; Zimonjic et al., 1995; Szuhai et al., 2000]. Ours is the first study that distinguishes these abnormalities from the other unselected rearrangements that are less likely to be directly relevant. It should be kept in mind that in the absence of constitutional karyotypes for the patients from whom the cell lines were isolated, it is not possible to be certain that any particular abnormality was somat- ically acquired, rather than representing a germ-line abnormality.

Our quantitative evidence of discordance between NCIN and S-CIN levels across the cell lines is consistent with findings in the relatively small range of other carcinoma types examined [Roschke et al., 2002; Gorringe et al., 2005; Bayani et al., 2008]. Our data suggest that different mechanisms underlie N-CIN and S-CIN in cervical SCC. The close association between modal chromosome number and $\mathrm{N}-\mathrm{CIN}$ is consistent with measures of 'karyotypic heterogeneity' in other carcinomas [Roschke et al., 2003]. The evidence suggests that the number of chromosomes in the cell may directly influence N-CIN rates, by determining how many chromosomes are avail- 
able to be gained or lost and/or the capacity of the cell to tolerate such copy number changes. In contrast, the absence of an association between modal chromosome number and S-CIN in cervical SCC is different from observations on karyotypic heterogeneity in other malignancies (mostly adenocarcinomas) [Roschke et al., 2003] and suggests that factors other than chromosome availability determine S-CIN levels.

Discordance between N-CIN and S-CIN in cervical SCC cells may have a number of potential explanations, including discrepant levels of HR-HPV E6 and E7 production [Duensing and Munger, 2002; Plug-DeMaggio et al., 2004]; differential responsiveness to E6 and/or E7 effects; and virus-independent host contributions. The latter may include, for example, efficiency of DNA double strand break repair [Someya et al., 2006], or production of transforming growth factor beta, which may contribute to telomere shortening by down-regulating c-myc, a rate-limiting protein in determining telomerase activity [Deng et al., 2008]. Moreover, the nature and relative importance of viral and host contributions to N-CIN and S-CIN may differ across the 8 cervical SCC cell lines. These issues deserve detailed, comprehensive investigation in a follow-up study. It is conceivable that in the future, identification of a predominant pattern of N-CIN or $\mathrm{S}$-CIN in clinical samples may suggest a specific treatment strategy directed at the cause, for example targeting DNA repair or mitotic pathways.

Despite evidence of ongoing S-CIN in all the cell lines, only 11 of the 118 selected abnormalities ( $\geq 70 \%$ metaphases) that we observed were absent from the lists of abnormalities seen in independent stocks of the cell lines by SKY/mCGH. This indicates that newly arisen rearrangements are infrequently selected in cervical SCC cells in vitro, supporting evidence for other carcinomas that selection is an important determinant of somatic evolution in malignancy [Tomlinson et al., 1996; Roschke et al., 2002; Gorringe et al., 2005]. It is likely that few abnormalities are selected in vitro as the cell environment is constant (selecting predominantly for growth); unlike in vivo, where a greater range and intensity of competitive pressures (e.g. host immunity, effects of chemo/radiotherapy) would be expected to lead to a faster rate of selection and clonal progression.

While the 11 abnormalities seen at high frequency by us and not by SKY would not have been present in the tumours from which the cell lines were derived, our data indicates that they do provide a selective advantage to cervical SCC cells in vitro. Such changes may still be of significance in cervical carcinogenesis, as karyotype ab-

Chromosomal Instability in Cervical SCC normalities that occur in cell culture can mirror those seen in independent clinical samples [Pett et al., 2004], presumably because the limited selective pressures that apply in vitro also pertain in vivo. The 38 rearrangements reported in the SKY/mCGH study but not seen by us (and therefore also likely to have been absent from the primary tumours) may also have included rearrangements that provided a strong selective advantage in vitro, although it is not possible to identify these without knowing the relative frequencies of the chromosomes seen in the SKY/ mCGH study [Harris et al., 2003].

Interestingly, we observed a consistent relationship between S-CIN levels and the numbers of clonal rearrangements in the cell lines, including a significant association between numbers of non-clonal and selected abnormalities. Exhaustive analysis of model systems (including overexpression of HPV16 E6 in epithelial cells) suggested that levels of S-CIN fluctuate during the early stages of neoplastic development, with a reciprocal relationship between numbers of clonal and non-clonal abnormalities (the latter defined using a $4 \%$ prevalence threshold) [Heng et al., 2006; Ye et al., 2009]. It is not known whether similar reciprocity occurs in early cervical carcinogenesis, where both HR-HPV oncogenes are expressed from the homologous viral promoter. However, our present findings suggest it is not a feature of established cervical SCC cells, similar to overall observations in other frankly malignant cells, mostly from adenocarcinomas [Roschke et al., 2003]. Importantly, our data argue that differences between cervical SCC cells in the number of rearrangements present are likely to reflect genuine biological differences affecting S-CIN, rather than being due to irrelevant confounding factors, such as length of time in culture, etc.

As chromosomal instability will cause ongoing karyotype changes in the SCC cell lines we have studied, we suggest that no one description can be considered definitive, particularly where the frequency of individual abnormalities is not provided. Because composite karyotypes, by definition, describe clonal abnormalities only, they provide limited information on karyotypic heterogeneity and cannot be used to infer prevailing rates of CIN. Our quantitative approach has allowed us to identify the abnormalities most relevant to cervical squamous carcinogenesis, which are therefore most deserving of further study. Moreover, our demonstration of dissociation of N-CIN and S-CIN levels in cervical SCC cells has implications for understanding mechanisms of neoplastic progression in HR-HPV-associated SCC and potentially in SCCs at other anatomical sites.

Cytogenet Genome Res 2009;127:9-20 


\section{References}

-Bayani J, Selvarajah S, Maire G, Vukovic B, AlRomaih K, et al: Genomic mechanisms and measurement of structural and numerical instability in cancer cells. Semin Cancer Biol 17:5-18 (2007).

-Bayani J, Paderova J, Murphy J, Rosen B, Zielenska M, Squire JA: Distinct patterns of structural and numerical chromosomal instability characterize sporadic ovarian cancer. Neoplasia 10:1057-1065 (2008).

Dall KL, Scarpini CG, Roberts I, Winder DM, Stanley MA, et al: Characterization of naturally occurring hpv16 integration sites isolated from cervical keratinocytes under noncompetitive conditions. Cancer Res 68: 8249-8259 (2008).

DDeng W, Tsao SW, Kwok YK, Wong E, Huang $\mathrm{XR}$, et al: Transforming growth factor beta 1 promotes chromosomal instability in human papillomavirus 16 E6E7-infected cervical epithelial cells. Cancer Res 68:7200-7209 (2008).

Duensing S, Munger K: The human papillomavirus type 16 E6 and E7 oncoproteins independently induce numerical and structural chromosome instability. Cancer Res 62: 7075-7082 (2002).

Duesberg P, Li R, Fabarius A, Hehlmann R: Aneuploidy and cancer: From correlation to causation. Contrib Microbiol 13:16-44 (2006).

-Garini Y, Macville M, du Manoir S, Buckwald RA, Lavi M, et al: Spectral karyotyping. Bioimaging 4:66-72 (1996).

-Gorringe KL, Chin SF, Pharoah P, Staines JM, Oliveira C, et al: Evidence that both genetic instability and selection contribute to the accumulation of chromosome alterations in cancer. Carcinogenesis 26:923-930 (2005).

- Harris CP, Lu XY, Narayan G, Singh B, Murty VV, Rao PH: Comprehensive molecular cytogenetic characterization of cervical cancer cell lines. Genes Chromosomes Cancer 36: 233-241 (2003).

Heim S, Mitelman F: Cancer Cytogenetics, 2nd ed (Wiley-Liss Inc, New York 1995).

- Heim S, Mandahl N, Mitelman F: Genetic convergence and divergence in tumor progression. Cancer Res 48:5911-5916 (1988).

-Heng HH, Bremer SW, Stevens J, Ye KJ, Miller F, et al: Cancer progression by non-clonal chromosome aberrations. J Cell Biochem 98: 1424-1435 (2006).
ISCN (2005): An International System for $\mathrm{Hu}-$ man Cytogenetic Nomenclature, Shaffer L, Tommerup N (eds). (S. Karger, Basel 2005).

James GK, Kalousek DK, Auersperg N: Karyotypic analysis of two related cervical carcinoma cell lines that contain human papillomavirus type 18 DNA and express divergent differentiation. Cancer Genet Cytogenet 38: 53-60 (1989).

Lengauer C, Kinzler KW, Vogelstein B: Genetic instability in colorectal cancers. Nature 386: 623-627 (1997).

Mitelman F, Johansson B, Mertens F: Catalog of Chromsome Aberrations in Cancer, 5th ed (Wiley-Liss, New York 1994).

-Morris M, Tortolero-Luna G, Malpica A, Baker VV, Cook E, et al: Cervical intraepithelial neoplasia and cervical cancer. Obstet Gynecol Clin North Am 23:347-410 (1996).

-Nacheva EP, Gribble S, Andrews K, Wienberg J, Grace CD: Screening for specific chromosome involvement in hematological malignancies using a set of 7 chromosome painting probes. An alternative approach for chromosome analysis using standard fish instrumentation. Cancer Genet Cytogenet 122:65-72 (2000).

Ng G, Winder D, Muralidhar B, Gooding E, Roberts I, et al: Gain and overexpression of the oncostatin $\mathrm{M}$ receptor occur frequently in cervical squamous cell carcinoma and are associated with adverse clinical outcome. Pathol 212:325-334 (2007).

- Pett M, Coleman N: Integration of high-risk human papillomavirus: A key event in cervical carcinogenesis? J Pathol 212:356-367 (2007).

- Pett MR, Alazawi WO, Roberts I, Dowen S, Smith DI, et al: Acquisition of high-level chromosomal instability is associated with integration of human papillomavirus type 16 in cervical keratinocytes. Cancer Res 64: 1359-1368 (2004).

$\rightarrow$ Plug-DeMaggio AW, Sundsvold T, Wurscher MA, Koop JI, Klingelhutz AJ, McDougall JK: Telomere erosion and chromosomal instability in cells expressing the HPV oncogene 16E6. Oncogene 23:3561-3571 (2004).

-Rajagopalan H, Nowak MA, Vogelstein B, Lengauer C: The significance of unstable chromosomes in colorectal cancer. Nat Rev Cancer 3:695-701 (2003).
Rao PH, Cigudosa JC, Ning Y, Calasanz MJ, Iida $S$, et al: Multicolor spectral karyotyping identifies new recurring breakpoints and translocations in multiple myeloma. Blood 92:1743-1748 (1998).

Roberts I, Wienberg J, Nacheva E, Grace C, Griffin D, Coleman N: Novel method for the production of multiple colour chromosome paints for use in karyotyping by fluorescence in situ hybridisation. Genes Chromosomes Cancer 25:241-250 (1999).

- Roschke AV, Stover K, Tonon G, Schaffer AA, Kirsch IR: Stable karyotypes in epithelial cancer cell lines despite high rates of ongoing structural and numerical chromosomal instability. Neoplasia 4:19-31 (2002).

- Roschke AV, Tonon G, Gehlhaus KS, McTyre N, Bussey KJ, et al: Karyotypic complexity of the NCI-60 drug-screening panel. Cancer Res 63:8634-8647 (2003).

Someya M, Sakata K, Matsumoto Y, Yamamoto $\mathrm{H}$, Monobe $\mathrm{M}$, et al: The association of DNAdependent protein kinase activity with chromosomal instability and risk of cancer. Carcinogenesis 27:117-122 (2006).

-Szuhai K, Bezrookove V, Wiegant J, Vrolijk J, Dirks RW, et al: Simultaneous molecular karyotyping and mapping of viral DNA integration sites by 25-color COBRA-FISH. Genes Chromosomes Cancer 28:92-97 (2000).

Tomlinson IP, Novelli MR, Bodmer WF: The mutation rate and cancer. Proc Natl Acad Sci USA 93:14800-14803 (1996).

Trent JM, Kaneko Y, Mitelman F: Report of the committee on structural chromosome changes in neoplasia. Cytogenet Cell Genet 51:533-562 (1989).

Ye CJ, Stevens JB, Liu G, Bremer SW, Jaiswal AS, et al: Genome based cell population heterogeneity promotes tumorigenicity: The evolutionary mechanism of cancer. J Cell Physiol 219:288-300 (2009).

Zimonjic DB, Simpson S, Popescu NC, DiPaolo JA: Molecular cytogenetics of human papillomavirus-negative cervical carcinoma cell lines. Cancer Genet Cytogenet 82:1-8

(1995).
zur Hausen H: Papillomaviruses causing cancer: Evasion from host-cell control in early events in carcinogenesis. J Natl Cancer Inst 92:690698 (2000). 